\title{
A Generic Method for Energy-Efficient and Energy-Cost-Effective Production at the Unit Process Level
}

Xu Gong*, Toon De Pessemier, Wout Joseph, Luc Martens

WiCa, Ghent University/iMinds, Gaston Crommenlaan 8 box 201, 9050 Ghent, Belgium.

* Corresponding author. E-mail: xu.gong@intec.ugent.be. Tel.: +32 933 14908. Fax: +32 93314899.

\begin{abstract}
Generally, industry includes various sectors like manufacturing, energy, materials \& mining, and transportation. Industry consumes about one half of the world's total delivered energy, and manufacturing is one of the energyintensive industrial sectors. With the rising energy price, the energy cost is becoming a controllable expenditure in manufacturing. In this paper, a generic method has been proposed to minimize the energy cost and improve the energy efficiency of manufacturing unit processes. Finite state machines have been used to build the transitional state-based energy model of a single machine. A mixed-integer linear programming mathematical model has been formulated for energy-cost-aware job order scheduling on a single machine. A generic algorithm has been implemented to search for an energy-cost-effective schedule at volatile energy prices with the constraint of due dates. As a result, plant managers can have an energy-cost-effective job order schedule which is associated with machine energy states along time, and can also get time-indexed energy simulation of the schedule. In comparison to most of the static scheduling approaches, stochasticity has been further handled through a cyclic interaction between the scheduler and the energy model, which facilitates to investigate how stochasticity on a shop floor affects the performance of energy-cost-aware scheduling. Empirical data have been used in the case study, including the power measured from a grinding machine, and the real-time pricing and time-of-use pricing tariffs. The proposed method has been demonstrated to be both energy-efficient and energy-cost-efficient even at the presence of stochasticity. As a joint effort of energy efficiency and demand response within demand side management, this method shows its effectiveness for contributing to the reduction of greenhouse gas emissions during peak periods, and for leading to energy-efficient, demand-responsive, and cost-effective manufacturing processes.
\end{abstract}

\section{Keywords}

Energy modeling Volatile energy price Sustainable production scheduling

Energy cost minimization $\quad$ Energy consumption forecast

\section{Introduction}

Traditionally, utilities called upon peak power generation to meet rising demand from energy consumers in a real-time manner. Those peak power generators were usually thermal power plants in high emissions of greenhouse gas (GHG). As a consequence, the stability of the power grid was threatened and the environment was seriously polluted. The demand side management (DSM) (Gelazanskas and Gamage, 2014), a set of interconnected and flexible programs including energy efficiency (EE) and demand response (DR), enables energy users of all types to highly take their own initiatives in maintaining the stability of the power grid. Environmental sustainability and economic saving are thus both achieved. As to industrial energy users, EE seeks approaches to reduce their energy consumption without declining the production outputs, while DR encourages a temporary change in their electricity consumption in response to market or supply conditions (Hadera et al., 2015). In summary, EE can be seen as load reduction, and DR can be viewed as load shift (Davito et al., 2010). Both EE and DR are among the major roadmaps to implement Smart Grid (Cardenas et al., 2014).

In order to make a balance between the power supply and demand during time, various energy charging policies are given in different countries, e.g., time-of-use pricing (ToUP), real-time pricing (RTP), and critical peak pricing (CPP). In ToUP tariff, two types of periods are generally defined: "on-peak" and "off-peak". The kWh energy 
charge during on-peak periods can be evidently higher than that during off-peak periods, such as more than twice (Babu and Ashok, 2008). RTP can be commonly found in countries whose energy market is highly developed. For instance, on "Belpex", the electricity spot market in Belgium (Belpex, 2014), users can buy a certain amount of electricity in two different submarkets, namely the day-ahead market (DAM) and the continuous intraday market (CIM). The DAM enables users to purchase electricity whose price is different every hour, and which will be delivered the day after. The CIM provides the industry with hourly-dynamic or multi-hourly-dynamic electricity up to five minutes before delivery. With an increasing amount of energy provision by volatile energy sources such as wind turbines, the RTP complies with the principle of demand and response. Time periods with surpluses of energy and low grid demands, result in low energy prices, while periods with only little energy from renewable energy sources and high grid demands, lead to high energy prices (Küster et al., 2013). Besides the non-event days during which the default ToUP is applied, CPP has mid-peak and critical periods on critical event days. During the two types of peak periods, the electricity price is set much higher, in order to reflect the marginal cost of electricity generation. For instance, in the Korean CPP pilot, the critical peak price and the mid-peak price are about 4.8 times and 3 times higher than the peak price and the off-peak price on non-event days, respectively (Jang et al., 2015).

The industry plays a key role in the society's overall energy consumption and GHG emissions. It thus exhibits a high potential for reducing both energy and GHG. For example, in Taiwan, the industrial energy consumption and GHG emissions account for approximately $53.8 \%$ and $48.3 \%$ by taking the entire country as a whole. The total energy saving in Taiwan's industry is assessed as $5.3 \%$ of the national energy use per year. The maximal GHG emissions reduction of Taiwan's six most energy-intensive industrial sectors is estimated as $6.4 \%$ of the national GHG emissions (Lu et al., 2013). Production activities are widely seen in the manufacturing industry (Chiarini, 2014). Therefore, it remains meaningful to investigate the energy consumption of production processes, in order to achieve better energy efficiency and energy cost (EC) efficiency in industry.

Under the scope of EE, the energy modeling of unit production processes does not consider the impact of volatile energy prices (see Section 2.1). In the scope of DR, the limited energy-cost-aware production scheduling researches tend to have weak capacities of modeling the energy consumption and of performing an effective scheduling according to dynamic energy prices (see Section 2.2). The conversion from energy consumption amount in $\mathrm{kWh}$ to energy consumption cost should be more explicit for decision-makers to get clear conscious of the economic benefit brought by energy consumption improvement measures. Therefore, a more advanced production scheduling algorithm should be developed, which is both energy aware and EC aware corresponding to EE and DR, respectively. Consequently, the industry is able to take advantage of lower-priced periods for extensive production or for storing energy for subsequent use during higher-priced periods.

In this paper, a generic method is proposed to perform energy modeling, simulation, and optimization for a unit manufacturing process. The novelty includes: (1) a joint connection of EE and DR is carried out to fully explore the industrial energy saving potentials within the DSM; (2) built on finite state machines (FSMs), the energy model is extensible and enables detailed energy simulation; (3) by using a genetic algorithm (GA), the energy-cost-aware scheduler assigns the job sequence such that electricity pricing peaks are avoided and valleys are took advantage of; (4) the power measurement on a surface grinding machine and two real dynamic electric tariffs fully demonstrate the applicability and effectiveness of the proposed method; (5) the energy consumption of a unit process can be forecasted according to the energy-cost-aware scheduling solution.

The rest of this paper is organized as follows. Section 2 provides a literature review revealing the problem. Section 3 proposes a generic method to solve the problem, which covers energy modeling and simulation, energycost-aware job scheduling, and GA-based EC minimization. Section 4 describes the implementation of this method in the case study of a surface grinding machine. Section 5 finally draws the related conclusions.

\section{Literature review}

The studied issues in this paper include energy modeling for a unit manufacturing process and energy-costaware scheduling of a single machine. The former research investigates how to increase the transparency of machine 
energy consumption, which paves the way for reducing energy consumption. Thereby, it is within the scope of EE. The latter takes variable energy prices into consideration and shifts the production along the time course such that low energy prices are made use of as many as possible. This is part of the principal measures taken by industrial end-users to implement DR. The rest of this section will discuss the state of the art in these two fields.

\subsection{Energy modeling for unit processes}

Prior to energy modeling, electrical energy metering in complex manufacturing facilities is necessary to provide industrial enterprises higher levels of quantification and visibility in their energy consumption. Both voltage and current need to be measured at either low or high sampling rates, in order to calculate power consumption and to produce more complex power quality statistics such as sags, peaks, and harmonics (O'Driscoll and O'Donnell, 2013; Shrouf and Miragliotta, 2015). An energy management framework can be further established to promote energy awareness in manufacturing processes (Vikhorev et al., 2013). On the basis of the measured power, empirical energy models can be built for estimating the energy consumption related to the production. The rest of this sub-section focuses on energy modeling at the level of unit process.

Gutowski et al. (2006) used an exergy framework to examine the energy requirement for a wide range of unit processes such as milling, injection molding, and grinding. Specific energy consumption (SEC) was defined to describe the energy needed for processing one unit of material. The process rate was demonstrated through empirical experiments as the key variable influencing the energy requirement of a unit process. This relatively early finding pointed out the complexity of industrial energy consumption, but there was no systematic approach to energy modeling and simulation.

Dietmair and Verl (2009b) built an energy model for single machines via discrete state chart and transitions between states. In their model, operational states are defined by the functionality a specific machine has, and each state is associated with an energy consumption profile. A stochastic extension of the model is further provided to complement its stochastic simulation capacity. To achieve a global energy consumption optimization, they proposed to adjust the process parameters related to each state, but they did not further demonstrate this proposition.

Diaz et al. (2011) conducted characterization on the energy consumption of milling machine tools during their use stage. The best fitted model is found with a $95 \%$ confidence level. It could then be used to estimate the total energy consumed during cutting. The effect of workpiece material on power demand was also studied. However, this empirical energy model was specifically for milling processes and no concrete energy saving measures were given.

In the framework of CO2PE! Initiative, the two energy estimation methodologies for unit processes proposed by Kellens et al. (2012a, 2012b) are screening approach and in-depth approach, respectively. The screening approach relies on publicly available data and engineering calculations for energy use. In the in-depth approach, different production modes are identified by the time study, and the power consumption for each mode is measured during the power study. The energy consumption of a unit process can then be estimated through multiplying the power by the duration of an operation. Energy or EC optimization is out of their scope.

An empirical energy modeling method was developed by Li et al. (2013) to predict energy consumption of unit processes. This industrial environment oriented method comprises four stages, namely design of experiments (DoE), physical experiments, statistical analysis, and model validation. The case study on an extrusion process proved its ability to accurately predict energy consumption of unit processes. Briefly, their work also focuses on energy modeling.

In the approach of Abele et al. (2012), power measurements are not necessarily needed. A single machine tool is described by several functional modules which further consist of various components. Within their Hardware-inthe-Loop-Simulation (HiL-Simulation), a physical machine controller is connected to the simulation model so that the programmable logic control (PLC) or numerical control (NC) signals, which contain power-on states, axis speeds, machine tool movement path, process operations, etc., are coupled with the functional modules and components to enable continuous energy simulation of a machine tool. In their case study of a coolant pump, various component configurations were tried to gain higher energy efficiency. 
In addition to estimating the machine energy requirement within the work of Abele et al. described above, Eberspächer et al. (2014) further developed the HiL-Simulation model for real-time monitoring of the energy demand of a machine and its functional modules in production environments. This energy monitoring system is claimed to raise the awareness of machine tool manufacturers and operators with regard to the machine energy consumption and to clearly show the consequences of their actions towards energy efficiency. Energy optimization measures based on components and operating states were finally discussed but not fully demonstrated.

\subsection{Energy-cost-aware production scheduling}

The traditional manual production schedule continuously becomes difficult in modern factories, where the production environment gets increasingly complicated. For instance, in semiconductor manufacturing, the dynamic job arrival, job re-circulation, shift bottlenecks, and lenghy fabrication process are all involed. A wafer brabrication process typically contains over 500 processing steps (Chien et al., 2012). Besides, multiple products can be produced by the same line (Ajorlou and Shams, 2013). The product variety is even increasing in order to satisfy the rapid changes at marketplaces (Huang et al., 2014). Furthermore, the volatile electricity price implies the need of frequent and short-term scheduling of plant operations, such as at a day-to-day time frame (Hadera et al., 2015). Therefore, it turns evident to foresee that an automated production scheduling is widely deployed in modern or future factories.

With energy monitoring systems increasingly implemented on shop floors, the transparency of production activities' energy consumption behaviors is improved. It is then feasible to add energy awareness to the conventional production schedulers which are part of manufacturing execution systems (MESs). The energy price can be further input into the scheduler, in order to facilitate its EC awareness.

Pechmann et al. (2012) developed production planning control (PPC) software that schedules the production on the basis of not only the usual planning criteria, i.e., deliver date, short lead-time, high resource utilization, and low inventory, but also their newly introduced objective of reducing peak power. The energy price was not explicitely considered, but a decrease of peak consumption was calculated to implicitely bring a cost reduction. As one of the key results given by this software, the 24-hour power load forecast for a plant has a 15-minute time step, which can only give a coarse estimation of energy consumption.

Küster et al. (2013) used multi-agent based distributed evolutionary algorithm to search for a multi-process schedule with an optimal EC. This approach makes use of the potential for rearranging process steps to shift loads to low-priced periods. However, they did not mention the details on machine energy consumption, i.e., the variable energy consumption along time, and the detailed machine startup/shutdown operations when encountering machine idle periods.

Fang and Lin (2013) combined energy consumption and tardiness as performance criteria for multi-machine scheduling. They proposed two heuristics respectively based on earliest due date (EDD) rule and weighted shortest processing time (WSPT) rule, and developed a particle swarm optimization (PSO). Nevertheless, both the energy consumption and EC were not clearly described. They simply assumed a higher machine speed would bring a shorter job makespan, while the corresponding energy consumption and EC would increase.

Luo et al. (2013) proposed a new ant colony optimization (MOACO) meta-heuristic taking into acount both makespan and electric cost to carry out hybrid flow shop scheduling. The ToUP mechanism and different machine processing speeds were considered. However, all the test data were randomly generated including the ToUP price and machine power consumption values. In addition, only two machine energy consumption states were assumed, i.e., processing and standby. The time aspect of scheduling results was unclearly described either.

Wang and Li (2013) tried to minimize respectively the electricity consumption and the electricity cost of manufacturing systems while respecting the production target. This problem was formulated and its near-optimal solution was searched by particle swarm optimization (PSO). The effects of the summer and winter ToUP pricing profiles on the scheduling result were also investigated. Nevertheless, machine transition states between off and producing, i.e., startup and shutdown, were ignored, and the power consumption value was theoretically assumed.

Zhang et al. (2014) adoped the ToUP tariff in their time-indexed integer programming formulation to conduct production scheduling. This scheduling minimizes electric cost while maintaining reasonable tradeoffs with 
production throughput and $\mathrm{CO}_{2}$ emission reduction, respetively. However, the concerned machines had only on and off modes, which turns out to be too simple for energy modeling. Furhermore, both energy profiles and ToUP tariff values were theoretically supposed.

Liu et al. (2014) built a bi-objective model and used Non-dominant Sorting Genertic Algorithm (NSGA-II) to minimize total energy consumption and total weighted tardiness on shop floors. However, only limited energy states were introduced in their energy model, i.e., idle, runtime, and cutting. Besides, the energy price was not considered to convert energy consumption into a more meaningful EC. He et al. (2015) optimized machine tool selection and operation sequence in job shops, in order to save energy consumption following the trade-off with makespan. Nonetheless, the volatile energy price was not taken into account, either. Liu et al. (2015) further formalized a mathematical model for the tri-objective job shop scheduling. By using NSGA, it reduces electricity consumption and cost while keeping good performance in total weighted tardiness, when the Rolling Blackout policy is applied. A trade-off was found between total weighted tardiness and total electricity cost. Nevertheless, both the involved energy model was simplified and the energy price was theretical.

Shrouf et al. (2014) used a GA to optimize the production scheduling of a single machine. Their schedule takes into account the dynamic electricity price to minimize the related electric cost. However, they only focused on determining when each job would start, and ignored scheduling the actual job sequence, which caused the job sequence on the same machine to be always fixed. Besides, they used a limited number of machine states, i.e., idle, processing, and shutdown, as well as presumed power values to model the energy consumption of a machine. This, together with the theoretical values for electricity price, caused a gap between their work and the industrial application.

Besides, stochasticity is a practical issue on a shop floor. Its occurrence can be and should be handled by the scheduler. Stochastic events (SEs) on a shop floor include machine failures (MFs), starvation or blockage of a production unit, cancellation or change of a customer order, etc. Each event has its corresponding statistical distribution to occur. For instance, a machine breakdown is often modeled by following the Weibull distribution (see Section 4.4). These events are seen as disturbance to a production schedule, since they interrupt the execution of the original schedule. A right-shift rescheduling policy (Cui et al., 2014; Liu et al., 2015), is often used to deal with similar situations: the originally scheduled job sequence stays unchanged, and the queuing jobs are postponed for an amount of time to just accommodate the SE duration.

\subsection{Summary}

Within the scope of EE, the energy models described in section 2.1 mainly aimed at estimating the power consumption profile of a machine. Very few efforts have been carried out to link energy modeling to energy-aware or energy-cost-aware production scheduling, for the purpose of effectively optimizing energy or cost consumption of a single machine. Within the scope of DR, according to the literature research in Section 2.2, there are currently limited studies on the optimization of manufacturing processes by considering variable energy prices. Very few energy aware or energy-cost-aware scheduling models have been built upon detailed energy models, in order to have an accurate estimation of energy or EC consumption. Furthermore, the aspect of energy modeling and simulation in those limited studies was weakly demonstrated.

\section{Method description}

The proposed method is described within the framework of DSM. It combines EE and DR, in comparison to most of the research which focuses on only one of the two domains. A generic energy modeling heuristic is introduced for EE in Section 3.1. Built on the generic energy model, an energy-cost-aware job scheduler and a GA optimization are proposed for DR, in Section 3.2 and Section 3.3, respectively. Section 3.4 further introduces a heuristic to generally handle stochasticity in the scheduling, in order to make the proposed method more adapted to the real factory environment. 


\subsection{Generic energy model}

The generic energy model for a single machine is described by the finite state machine (FSM). The FSM is commonly used to represent discrete events and logic systems. It comprises five basic elements: a set of states, state transitions, external inputs, initial state, and final state. It can be depicted by either formulas or graphs. The graphical representation is preferred herein, since it is more intuitive.

Only the normal energy consumption mode is considered in this generic model. The energy saving mode is out of scope, as there are currently a rather limited number of machines supporting this functionality (Weinert and Mose, 2014). As presented in Figure 1, the generic FSM energy model consists of four main states: (1) Off, (2) Startup, (3) Unowned, and (4) Shutdown. The composite state Unowned indicates that no energy management policy is owned by the machine. It further contains two sub-states: Ready and Production.

The initial state of each simulation is Off. It indicates the machine is powered off and consumes no energy. Upon receiving the event "Power on", the state transition is triggered from Off to Startup. At Startup, the turn-on of machine sub-units is carried out in a consecutive manner instead of turning on all sub-units at one time. This complies with the measured startup energy profiles of different production machines (Devoldere et al., 2008; Dietmair and Verl, 2009a). Following the completion of turn-on operation, the machine updates its status list, which contains the power on/off state of each sub-unit. This self-transition continues until all the sub-units are turned on and the Boolean signal "Ready" becomes true.

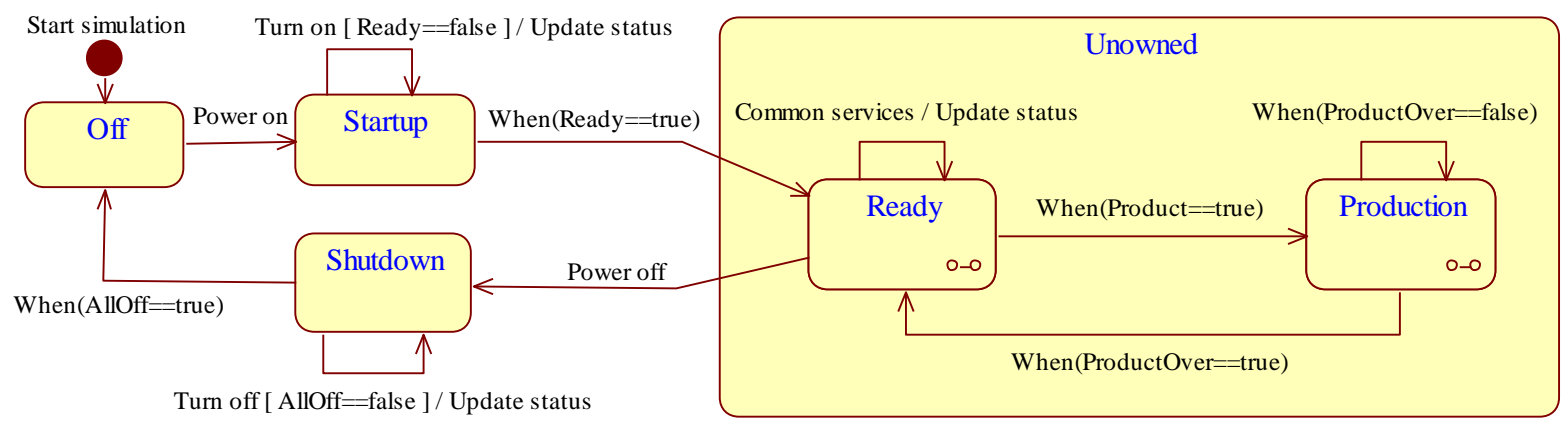

Figure 1 Industrial energy model at machine layer

Triggered by the "Ready" signal which turns true, the machine passes to the composite state Unowned. The entrance sub-state of Unowned is Ready, signifying that the machine is ready for production. The signal event "Common services" triggers a self-transition at Ready. The self-transition terminates by updating the machine status. "Common services" are to be defined according to the case study, e.g., to check the input material's availability. Once a production schedule is given, the Boolean signal "Product" changes from false to true. This then triggers the state transition to Production. The machine stays at this state until it completes the current production. When the signal event "ProductionOver" becomes true, it triggers the state transition back to Ready.

The signal event "Turn off" occurring at Ready triggers the state transition towards Shutdown. At Shutdown, the machine turns off its sub-units also in a consecutive manner. This continues until the machine updates its status list such that all the sub-units are powered off and the Boolean signal "AllOff" becomes true. This finally drives the machine back to Off. The final state of each simulation is by default set as Off. Upon a SE, a simulation run terminates at any one of the states (see Section 3.4).

Based on the measured power consumption, different energy consumption states can be identified. The state identification method is similar to the time study of the in-depth approach proposed by Kellens, Dewulf, Overcash, Hauschild and Duflou (2012a). So the time span of each state can be determined. Exceptionally, the duration of Ready can be arbitrary, as it is a state for staying idle. In the model proposed by this paper, two types of Ready durations are thus defined, i.e., default duration and customized duration. The default duration for Ready stands for the necessary internal machine time for an immediate transition from Startup to Production, or from Production to 
Shutdown. In comparison, the customized duration is fixed by the production schedule, which can be an arbitrary value not less than the default duration.

Furthermore, a mean power is associated with each machine state. The machine energy consumption $E$ during a simulation can thus be estimated by Equation (1):

$$
E=\sum_{s \in S} \sum_{t \in T_{s}} P_{s} \cdot t
$$

where $s$ is a machine state, $S$ is the set of machine states, $t$ is a time period during which the machine stays at the state $s, T_{s}$ is the set of periods during which the machine stays at the state $s$, and $P_{s}$ is the mean power consumption of the state $s$. This complies with the energy modeling approach of Mechs et al. (2012). This general mapping enables a quick denotation of the fundamental energetic performance. Moreover, based on the identified energy profiles of machine states, the energy model can be further developed to provide machine energy KPIs and energy consumption details.

\subsection{Energy-cost-aware job scheduler}

With the consideration of the volatile electricity price, the proposed scheduler aims to assign the job sequence and machine states, such that all the jobs allocated to this machine contribute to a minimal electric cost without missing the same required due time. No interdependence is assumed to exist among jobs so an arbitrary job sequence can be generated. This job scheduler is a discrete-time system, since it is built upon the FSM energy model. Its basic time step is quite flexible depending on the applied case, especially on the frequency of the measured energy data injected into the energy model. The inputs of this scheduler are variable electricity prices, job IDs and production durations, a pre-fixed due time, and the energy model introduced in Section 3.1. The outputs include the job sequence, the start time and end time of each job, the machine operation following the completion of each job, and also a detailed energy and cost audit for the current scheduling solution. The machine operation can be "immediately start the next job", "shut down", or "stay idle".

\begin{tabular}{c|c} 
Table 1 Numeration for machine states \\
\hline Machine state $s$ & Index \\
\hline Off & 1 \\
Startup & 2 \\
Ready & 3 \\
Production & 4 \\
Shutdown & 5 \\
Others & $\ldots$ \\
\hline
\end{tabular}

A mathematical model is formulated below for this problem. The concerned parameters are first introduced. Then the objective function is given, followed by a bunch of relations or constraints. For the sake of conciseness, each machine state is assigned a unique integer index. As shown in Table 1, the last item "others" is specially retained for any case study that needs to extend the generic FSM energy model.

Equation (2) is the objective function which sets machine states and the job sequence along time such that the electric cost for conducting all the jobs within the concerned work shifts is minimal. Equation (3) calculates the electric cost for completing a scheduled job. Equation (4) calculates the electric cost for the machine to stay at Ready between job $j$ and the next scheduled job. Equation (5) obtains the electric cost for the machine to be shut down between job $j$ and the next scheduled job. The concerned cost can be further cut into three parts: cost for staying at Ready during a default duration, cost for shutting down, and cost for starting up the machine after staying powered off and just before the beginning of the next scheduled job. Equation (6) determines the machine to stay at Ready if there is not enough time between two adjacent scheduled jobs for the machine to be shut down, as the durations of powering off and powering on again the machine are implicitly included, or if the cost for powering off is more expensive.

Equation (7) requires the duration of the first scheduled job comprises the time for the machine to start up, pass by Ready for the default duration, and execute the job. Equation (8) defines that the duration of an intermediate job should consist of a default duration of Ready at the beginning and then the job execution time. The default Ready 
duration before the actual job execution is considered as necessary machine time to receive and read the next production schedule. Equation (9) ensures the duration of the last scheduled job to include the default duration of Ready, the job execution time, the default duration for the machine to pass by Ready, and finally the time span for shutting down. Equation (10) guarantees each job is scheduled only once and thus all the jobs can be scheduled. Equation (11) limits the machine can have only one state at one point of time. Equation (12) uses the flooring function to decide at which pricing slot the discrete time is located. Equation (13) calculates the duration for staying powered off between two jobs. Equation (14) makes sure that only one job is executed at one time on respecting the scheduled job sequence, and pre-emption is prohibited. Equation (15) shows the requirement that there is enough time to fulfill all the jobs and the machine shutdown before the due time.

\section{Parameters}

$C j$ : electric cost for the $j$ th scheduled job, $j \in\left[1,2, \ldots, N_{J}\right]$.

$C R_{j}$ : electric cost for the machine to stay at Ready state after the completion of the $j$ th job, $j \in\left[1,2, \ldots, N_{J^{-}} 1\right]$.

$C S D_{j}$ : electric cost for the machine to be shut down after the completion of the $j$ th job, $j \in\left[1,2, \ldots, N_{J}-1\right]$.

$D$ : time duration of one pricing slot.

$D_{j}$ : processing duration in seconds for the job with ID $j, j \in\left[1,2, \ldots, N_{J}\right]$.

$D_{j}^{i}$ : processing duration in seconds for the job with ID $j$ at the $i$ th scheduling position, $i$ and $j \in\left[1,2, \ldots, N_{J}\right]$. A job ID never changes once assigned, whereas the scheduling position of a job can be variable in different job schedules.

DT: due time for all the jobs in the concerned work shifts.

$E P_{t s}$ : electricity price during the $t$ sth pricing time slot.

$E T_{j}$ : end time for the $j$ th scheduled job, $j \in\left[1,2, \ldots, N_{J}\right]$.

$E T S_{j}$ : end time in slots for the $j$ th scheduled job, $j \in\left[1,2, \ldots, N_{J}\right]$.

$N_{J}$ : total number of jobs in the concerned work shifts.

$N_{s}$ : total number of machine states.

$P_{s}$ : power consumption of the machine state $s$.

$P_{s}^{t}$ : power consumption of machine state $s$ at time $t$. It equals to $P_{s}$ when the machine state at $t$ is $s$; otherwise zero.

$S T_{j}$ : start time for the $j$ th scheduled job, $j \in\left[1,2, \ldots, N_{J}\right]$.

$S T S_{j}$ : start time in slots for the $j$ th scheduled job, $j \in\left[1,2, \ldots, N_{J}\right]$.

$T_{s}$ : start time of the concerned work shifts.

$T O$ : time duration for the machine to stay off.

$T R$ : default time duration for the machine to stay ready. Compared to the duration to stay idle during which the machine is also at Ready state, it is viewed as internal machine time to receive and read the next job schedule.

$T S D$ : time duration to shut down the machine.

$T S U$ : time duration to start up the machine.

$s:$ machine state, $s \in\left[1,2, \ldots, N_{s}\right]$.

$t$ : time in the defined basic unit, $t \in[0, \ldots, \mathrm{DT}]$.

$t s$ : time in electric pricing slots, $t s \in[1,2, \ldots$, ceil(DT/D)]. The function ceil() rounds the value toward positive infinity.

$\alpha_{j}$ : machine operation indicator. When the current job is completed, if the machine is set to stay at Ready state,

$\alpha_{j}$ is one; if the machine is set to be shut down, $\alpha_{j}$ is zero, $j \in\left[1,2, \ldots, N_{J}-1\right]$.

$\beta_{t s}$ : time reference factor. If $t$ is in the $t$ sth time slot, $\beta_{t s}$ equals to one; otherwise zero.

$$
\min _{s, t}\left\{\sum_{j=1}^{N_{J}} C_{j}+\sum_{j=1}^{N_{J}-1}\left[\alpha_{j} \cdot C R_{j}+\left(1-\alpha_{j}\right) \cdot C S D_{j}\right]\right\}
$$

Subject to: 


$$
\begin{aligned}
& C_{j}=\sum_{t s=S T S_{j}}^{E T S_{j}} E P_{t s} \cdot\left(\beta_{t s} \cdot \sum_{t=S T_{j}}^{E T_{j}} \sum_{s=1}^{N_{s}} P_{s}^{t} \cdot t\right), j \in\left[1,2, \ldots, N_{J}\right] \\
& C R_{j}=\sum_{t s=E T S_{j}}^{S T S_{j+1}} E P_{t s} \cdot\left(\beta_{t s} \cdot \sum_{t=E T_{j}}^{S T_{j+1}} P_{3} \cdot t\right), j \in\left[1,2, \ldots, N_{J}-1\right] \\
& C S D_{j}=\sum_{t s=E T S_{j}}^{\left.\mid\left(E T_{j}+D_{3}-T_{S}\right) / D\right\rfloor} E P_{t s} \cdot\left(\beta_{t s} \cdot \sum_{t=E T_{j}}^{E T_{j}+D_{3}} P_{3} \cdot t\right)+\sum_{t s=\left[\left(E T_{j}+D_{3}-T_{S}\right) / D\right]}^{\left.\mid\left(E T_{j}+D_{3}+D_{5}-T_{S}\right) / D\right\rfloor} E P_{t s} \cdot\left(\beta_{t s} \cdot \sum_{t=E T_{j}+D_{3}}^{E T_{j}+D_{3}+D_{5}} P_{5} \cdot t\right)+ \\
& \sum_{t s=\left\lfloor\left(E T_{j}+D_{3}+D_{5}+T O-T_{s}\right) / D\right\rfloor}^{\left.\mid\left(E T_{j}+D_{3}+D_{5}+T O+D_{2}-T_{s}\right) / D\right\rfloor} E P_{t s} \cdot\left(\beta_{t s} \cdot \sum_{t=E T_{j}+D_{3}+D_{5}+T O}^{E T_{j}+D_{3}+D_{5}+T O+D_{2}} P_{2} \cdot t\right), j \in\left[1,2, \ldots, N_{J}-1\right] \\
& \alpha_{j}=\left\{\begin{array}{l}
1, \text { if }\left(S T_{j+1}-E T_{j}\right) \leq\left(D_{3}+D_{5}+D_{2}\right) \text { or } C R_{j} \leq C S D_{j} \\
0, \text { otherwise }
\end{array}\right. \\
& E T_{1}=S T_{1}+T S U+T R+D_{j}^{1}, j \in\left[1,2, \ldots, N_{J}\right] \\
& E T_{i}=S T_{i}+T R+D_{j}^{i}, i \in\left[2,3, \ldots, N_{J}-1\right], j \in\left[1,2, \ldots, N_{J}\right] \\
& E T_{N_{J}}=S T_{N_{J}}+T R+D_{j}^{N_{J}}+T R+T S D, j \in\left[1,2, \ldots, N_{J}\right] \\
& D_{j}=\sum_{k=1}^{N_{J}} D_{j}^{k}, j \in\left[1,2, \ldots, N_{J}\right] \\
& P_{s}^{t}=P_{s}=\sum_{k=1}^{N_{s}} P_{k}^{t}, s \in\left[1,2, \ldots, N_{s}\right] \\
& t s=\left\lfloor\left(t-T_{s}\right) / D\right\rfloor \\
& T O=\left\{\begin{array}{l}
0, \text { if } S T_{j+1}-E T_{j} \leq D_{3}+D_{5}+D_{2} \\
S T_{j+1}-\left(E T_{j}+D_{3}+D_{5}+D_{2}\right), \text { otherwise }
\end{array}, j \in\left[1,2, \ldots, N_{J}-1\right]\right. \\
& S T_{j}<E T_{j}, E T_{i}+T R \leq S T_{i+1}, j \in\left[1,2, \ldots, N_{J}\right], i \in\left[1,2, \ldots, N_{J}-1\right] \\
& E T_{N_{J}}+T R+T S D \leq D T
\end{aligned}
$$

\subsection{Electric cost minimization with a genetic algorithm (GA)}

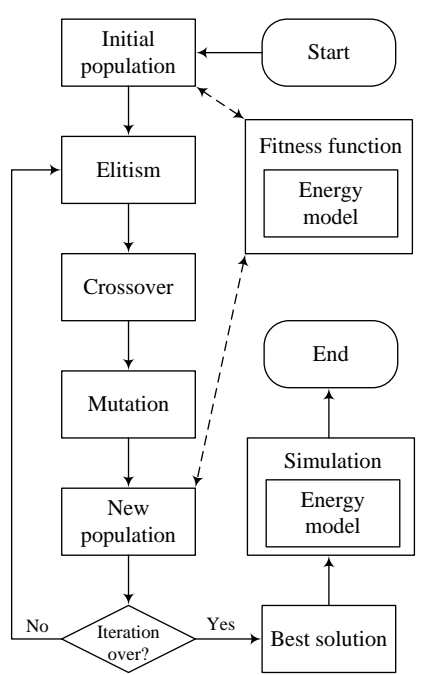

Figure 2 Implementation of a GA in the proposed method 
As presented in Figure 2, a genetic algorithm (GA) is used in the proposed method to search for the optimal solution to the scheduling problem formulated above, i.e., Equation (2) - (15). A gene contains the information of a certain job including job ID, job duration, workpiece number, job start time, EC for executing this job, and idle/off machine operation after the completion of this job. A chromosome is a complete scheduling solution including the job sequence, all the jobs with their detailed information, and the machine operation following each job. The crossover and mutation are two important operations on genes in a GA, on which the GA performance largely depends. The crossover creates child solutions from parent chromosomes. The mutation prevents falling all solutions into a local optimum of the solved problem. Besides, the elitism is implemented to ensure the best solutions of a generation can be always retained into the next generation. The fitness function (see Figure 2) containing the energy model evaluates each solution within a population. Therefore, a solution is the input of the energy model. The output is the electric cost for the input solution, which is further stored as the solution's fitness. When the maximal iteration number is reached, the best solution is selected and simulated which finally provides detailed energy simulation information.

\subsection{Stochasticity handling in energy-cost-aware scheduling}

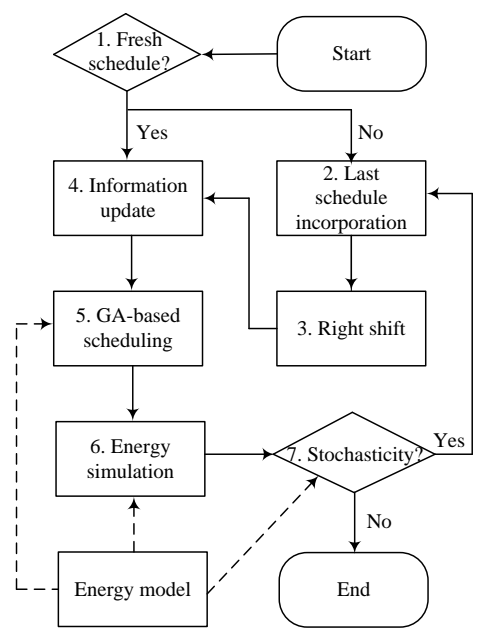

Figure 3 General handling of stochasticity in the scheduling

The proposed heuristic to handle stochasticity in the scheduling is presented in Figure 3. The key operations are indicated by different numbers. (1) The fresh schedule operation decides whether the next scheduling is run on the basis of a former schedule. In the case of stochasticity, a former schedule is the one that is interrupted by a SE. (2) If a former schedule is involved, the interrupted schedule is taken into consideration for the next scheduling. The considered information includes a) the time when the former schedule is interrupted by a SE (i.e., the start time of the SE), b) the duration of the SE, c) the already executed jobs in the former schedule, or the non-executed jobs that need to be reconsidered in the next scheduling, and d) the job that is being executed, but is not yet accomplished, upon the occurrence of the last SE.

(3) The next scheduling (i.e., rescheduling) can be started from the time when the last SE terminates (i.e., the start time plus the duration of the SE). The right-shift operation postpones all the upcoming jobs after the termination of the SE. Depending on the specific production, an interrupted job has to be totally reproduced (i.e., a non-resumable job), or only its non-executed part remains to be produced during the next schedule (i.e., a resumable job). So for an interrupted non-resumable job, its whole part is right-shifted. For an interrupted resumable job, its non-executed part is right-shifted. In comparison to the existing right-shift policy, rescheduling of job orders with the volatile energy price is involved in the following steps, in order to remain energy-cost-effective.

(4) Input information is updated and loaded in the scheduler, e.g., the energy price, input jobs, the start time, the due time, GA configurations, etc. (5) The scheduling is carried out by using the GA. (6) For the output optimal 
schedule, an energy simulation is conducted to have a detailed energy report of this schedule. (7) If stochasticity is involved, it will invoke another rescheduling. Otherwise, the whole procedure terminates.

The energy model is coupled with operation5 (O5) and $\mathrm{O} 6$ to make the scheduling and modeling energy-aware. It is also associated with $\mathrm{O} 7$ to incorporate stochasticity into its time progression. The sequential steps "Start-O1O4-O5-O6-O7-End" form up a conventional scheduling procedure, which is static. The cyclic steps "O2-O3-O4-O5O6-O7-O2-O3..." set up a dynamic scheduling procedure to deal with SEs.

\section{Case study}

The proposed method was implemented in a case study of a surface grinding machine (Paragon RC-18CNC) under two real electric pricing mechanisms (RTP and ToUP). The stochasticity handling in energy-cost aware scheduling was also investigated.

\subsection{Energy modeling of a single machine}

The power measurement on this grinder was performed with a clamp-on power meter (Yokogawa CW240). Connected between the power supply and the grinder, the power meter records the grinder's overall power consumption every second. The grinder's main energy consumers are listed in Table 2.

Table 2 Energy consumers of the grinding machine RC-18CNC

\begin{tabular}{ll}
\hline Energy consuming units & Function \\
\hline Grinding wheel & $\begin{array}{l}\text { Grind the workpiece (Each grain of abrasive on the grinding wheel's surface cuts } \\
\text { a small chip from the workpiece via shear deformation) } \\
\text { Regulating wheel }\end{array}$ \\
$\begin{array}{l}\text { Rotate the workpiece and pull it through the operation so as to control workpiece } \\
\text { rotational speed and feed rate } \\
\text { Tydraulic pump } \\
\begin{array}{l}\text { Toolant pump } \\
\text { Others (computer, light, } \\
\text { hydraulic oil cooler, } \\
\text { automatic lubricator etc.) }\end{array}\end{array}$ & Move coolant for cooling the workpiece, grinding wheel and regulating wheel \\
\end{tabular}

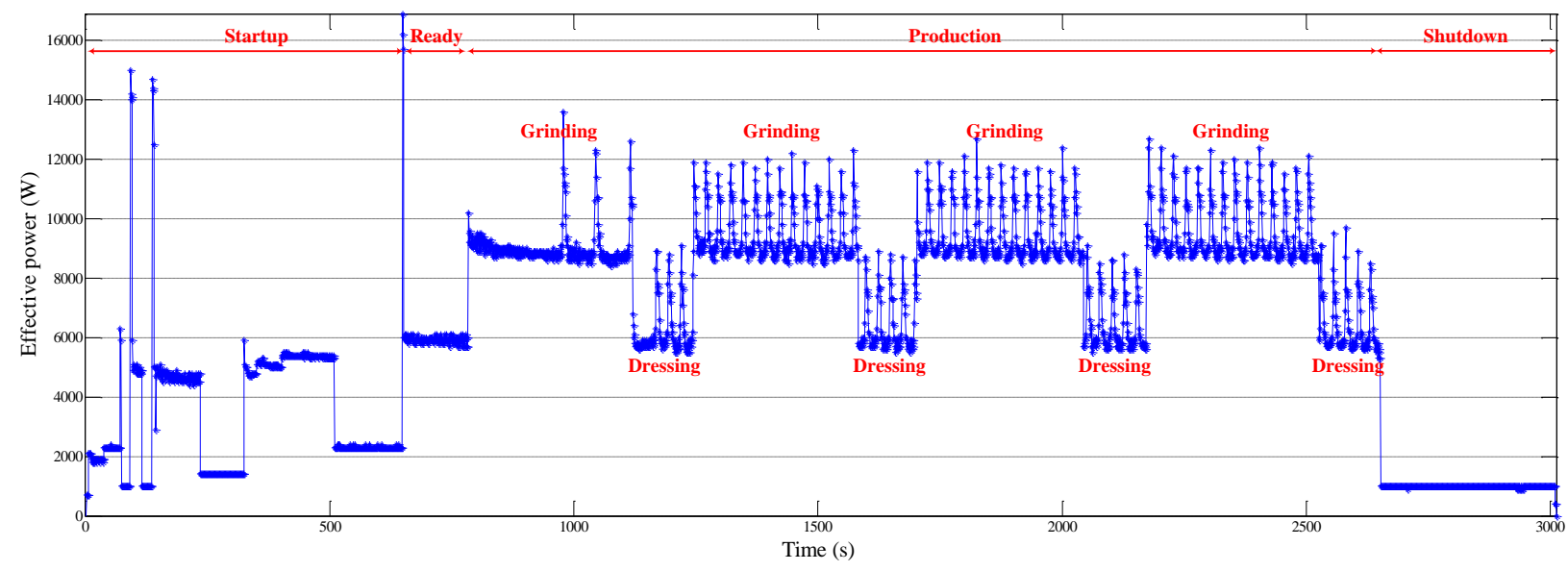

Figure 4 A complete energy consumption profile of the investigated grinding machine

This grinder's complete energy profile is illustrated in Figure 4. At Startup state, its power consumption first has sharp peaks at around $15 \mathrm{~kW}$, and then experiences a periodic drop-down and rise-up between $4.8 \mathrm{~kW}$ and $2 \mathrm{~kW}$, which should be due to the power-off and power-on of the first coolant pump. At Ready state, the grinding wheel rotates at a fixed peripheral speed of $2000 \mathrm{~m} / \mathrm{min}$ without the touch of a workpiece or the dresser, which results a 
nearly constant power consumption of $6 \mathrm{~kW}$. The Production state is further divided into Grinding and Dressing sub-states. Dressing is responsible for sharpening and regularizing the grinding wheel shape, and cleaning the impurities coming from the chips. The second coolant pump should turn on when the state transitions from Ready to Grinding. At Grinding, each evident peak corresponds to grinding one workpiece. The grinder passes from Grinding to Dressing about every 350 seconds. The second coolant pump should be turned off during the dressing cycle. At Shutdown, the main power consumers are powered off rapidly, which leads to the chute of the power curve; then the grinder stays in a constant power level for more than five minutes (see Figure 4). As the grinder is computernumerically controlled, this is interpreted as a compulsory duration for the numerical system to perform shutdown work, e.g., storing data to non-volatile memory. The energy audit for the grinder states is listed in Table 3. Some states have an obvious difference between their maximum and average powers, e.g., Startup and Shutdown, while others have steady power profiles, e.g., Ready.

Table 3 Energy audit for the grinder states

\begin{tabular}{lllll}
\hline $\begin{array}{l}\text { Machine state } \\
\text { (one cycle) }\end{array}$ & $\begin{array}{l}\text { Maximum power } \\
(\mathrm{kW})\end{array}$ & $\begin{array}{l}\text { Average power } \\
(\mathrm{kW})\end{array}$ & Duration (s) & $\begin{array}{l}\text { Average energy } \\
\text { consumed (kWh) }\end{array}$ \\
\hline Startup & 16.90 & 3.55 & 652 & 0.64 \\
Ready & 6.10 & 5.93 & 25 (default) & 0.04 (default) \\
Grinding & 12.07 & 9.49 & 25 & 0.07 \\
Dressing & 8.95 & 6.72 & 125 & 0.23 \\
Shutdown & 5.30 & 1.00 & 362 & 0.10 \\
\hline
\end{tabular}

Based on the identified states, the general energy model proposed in Section 3.1 was extended to be applied to this specific case, as shown in Figure 5. It was implemented in Java. Compared to the generic model, the Production state further contains Grinding and Dressing. Although the actual grinding operation has to be interrupted periodically, the dressing operation should be carried out not only to avoid the occurrence of abnormalities on the grinding wheel's surface but also to guarantee high product quality. The dresser is assumed to be in good condition when the grinder starts a new job. So when the machine stays at Ready, its next state is either Grinding or Shutdown.

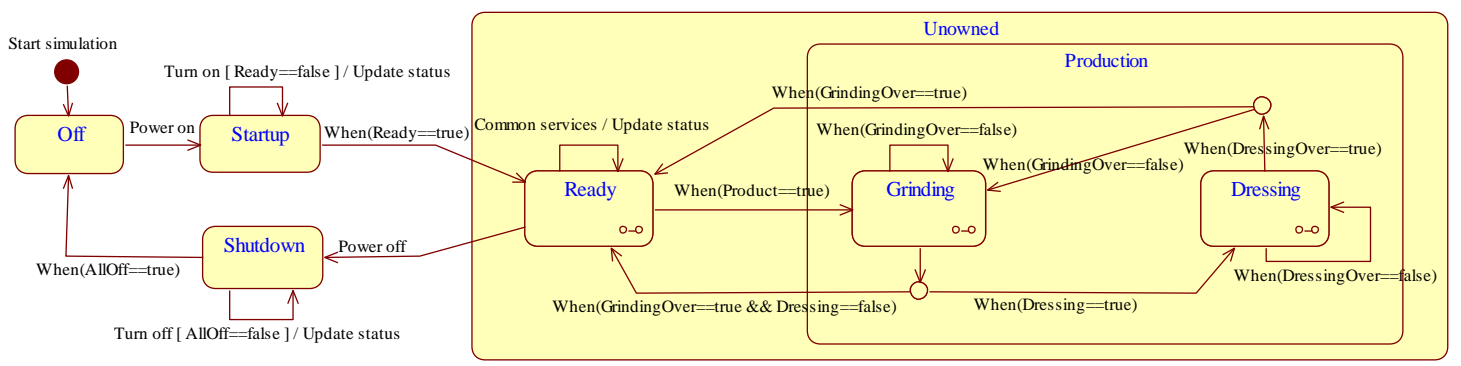

Figure 5 Specific energy model for surface grinding process

\subsection{Single-machine job scheduling under real-time pricing (RTP)}

The energy-cost-aware job scheduling model is expected to work such that the total electricity cost for the scheduled production is minimized under the dynamic pricing mechanism. It is coupled with the energy model built in Section 4.1. Therefore, it can not only get full knowledge of the energy-related information, but also output a scheduling solution for the energy-related simulation.

\subsubsection{Genetic algorithm (GA) optimization}

A number of assumptions were first made. (1) The concerned work shifts last from 8 am on March-3-2014 to 2 pm on March-4-2014. (2) At Belpex, since the exchanged power volume on DAM is significantly greater than that on CIM (Belpex, 2013), the RTP data is taken from DAM (see Figure 6). (3) The concerned steel workpieces are of the same type as that in the measurement. (4) The grinder runs the same numerical control (NC) program, which means it keeps the same energy consumption behavior as that identified in Section 4.1. Moreover, the machine 
always grinds 14 workpieces then conducts one cycle of dressing operation during a continuous grinding process. (5) If the machine grinds less than 14 workpieces just before it fulfills the current job, it will grind another 14 workpieces for the next job before it carries out another dressing operation. This is denoted as "non-memory dressing". (6) If the grinder stays idle or off before the start of one job, the start time of this job is always set at the very start of a certain hour, e.g., 9 am and $11 \mathrm{pm}$. (7) The grinding jobs are shown in Table 4. Totally five jobs are considered to take an example. An extension to a larger number of jobs is possible.

Table 4 Grinding jobs for scheduling

\begin{tabular}{lccccc}
\hline \multicolumn{1}{c}{ Job ID } & 1 & 2 & 3 & 4 & 5 \\
\hline Number of steel workpieces & 100 & 200 & 300 & 400 & 500 \\
Required production time in & 3375 & 6750 & 10125 & 13500 & 16875 \\
seconds (grinding + dressing) & $(56 \mathrm{~m} 15 \mathrm{~s})$ & $(1 \mathrm{~h} 52 \mathrm{~m} 30 \mathrm{~s})$ & $(2 \mathrm{~h} 48 \mathrm{~m} 45 \mathrm{~s})$ & $(3 \mathrm{~h} 45 \mathrm{~m})$ & $(4 \mathrm{~h} 41 \mathrm{~m} 15 \mathrm{~s})$ \\
\hline
\end{tabular}

Concerning the genetic algorithm (GA) implementation in Java, permutation encoding was used for crossover and mutation. Supposing there are two solutions represented by different orders of job IDs, i.e., solution1 is (2, 4, 1, $3,5)$ and solution 2 is $(5,1,4,2,3)$, the single-point crossover and mutation are then defined in Table 5 and Table 6 , respectively. Different to the conventional crossover rules, genes of two chromosomes are herein exchanged while ensuring that there is no job ID repetition in each chromosome (i.e., solution). For instance, in the possible case 1 in Table 5, job2 in child solution1 comes from solution1, and the rest jobs in child solution1 come from solution2 by following the job order in solution 2 while skipping job2 in solution2. The population size was set as 80 . This means that each generation has 80 individuals. The elitism rate was 0.15 , which means the top $15 \%$ of individuals were retained from one generation to the next. The crossover and mutation rates were fixed at $95 \%$ and $3 \%$, respectively. The maximal iteration was 100 .

\begin{tabular}{cllcll}
\multicolumn{2}{l}{ Table 5 Defined crossover } & \multicolumn{5}{l}{} \\
\hline Possible case & Solution1 & Solution2 & Crossover point & Child solution1 & Child solution2 \\
\hline 1 & $(\mathbf{2}, 4,1,3,5)$ & $(\mathbf{5}, 1,4,2,3)$ & 1 & $(\mathbf{2}, 5,1,4,3)$ & $(\mathbf{5}, 2,4,1,3)$ \\
2 & $(\mathbf{2}, \mathbf{4}, 1,3,5)$ & $(\mathbf{5}, \mathbf{1}, 4,2,3)$ & 2 & $(\mathbf{2}, \mathbf{4}, 5,1,3)$ & $(\mathbf{5}, \mathbf{1}, 2,4,3)$ \\
3 & $(\mathbf{2}, \mathbf{4}, \mathbf{1}, 3,5)$ & $(\mathbf{5}, \mathbf{1}, \mathbf{4}, 2,3)$ & 3 & $(\mathbf{2}, \mathbf{4}, \mathbf{1}, 5,3)$ & $(\mathbf{5}, \mathbf{1}, \mathbf{4}, 2,3)$ \\
4 & $(\mathbf{2}, \mathbf{4}, \mathbf{1}, \mathbf{3}, 5)$ & $(\mathbf{5}, \mathbf{1}, \mathbf{4}, \mathbf{2}, 3)$ & 4 & $(\mathbf{2}, \mathbf{4}, \mathbf{1}, \mathbf{3}, \mathbf{5})$ & $(\mathbf{5}, \mathbf{1}, \mathbf{4}, \mathbf{2}, 3)$ \\
\hline
\end{tabular}

\begin{tabular}{cll} 
Table 6 Defined mutation & \\
\hline Possible case & Solution & Mutated solution \\
\hline 1 & $(\mathbf{2}, 4, \mathbf{1}, 3,5)$ & $(\mathbf{1}, 4, \mathbf{2}, 3,5)$ \\
2 & $(2, \mathbf{4}, 1,3, \mathbf{5})$ & $(2, \mathbf{5}, 1,3, \mathbf{4})$ \\
Others & $\ldots$ & $\ldots$ \\
\hline
\end{tabular}

Table 7 Optimal job schedule for electric cost minimization

\begin{tabular}{|c|c|c|c|c|}
\hline Job ID & $\begin{array}{l}\text { Job start time } \\
(\text { March 2014) }\end{array}$ & $\begin{array}{l}\text { Job end time } \\
\text { (March 2014) }\end{array}$ & $\begin{array}{l}\text { Machine operation following } \\
\text { the current job }\end{array}$ & $\begin{array}{l}\text { Machine states following the } \\
\text { current job }\end{array}$ \\
\hline 3 & 3d:15h:0m:0s & 3d:18h:0m:2s & Immediately start the next job & Ready, Grinding + Dressing \\
\hline 1 & $3 \mathrm{~d}: 18 \mathrm{~h}: 0 \mathrm{~m}: 2 \mathrm{~s}$ & $3 \mathrm{~d}: 18 \mathrm{~h}: 56 \mathrm{~m}: 42 \mathrm{~s}$ & Shut down & Ready, Shutdown, Off \\
\hline 4 & 3d:21h:0m:0s & 4d:0h:45m:25s & Immediately start the next job & Ready, Grinding + Dressing \\
\hline 5 & 4d:0h:45m:25s & 4d:5h:27m:5s & Immediately start the next job & Ready, Grinding + Dressing \\
\hline 2 & 4d:5h:27m:5s & 4d:7h:26m:27s & Shut down & Ready, Shutdown, Off \\
\hline
\end{tabular}

The optimal job schedule found by the GA is shown in Table 7. The time step in this schedule is one second, since the measured power data has a frequency of one hertz. The scheduler's stability is proven by the fact that there 
is no time overlap between jobs, and in the case of consecutive jobs, the next job strictly starts from the end time point of its precedent job. The machine operation is also given to indicate the machine behavior following each job.

This schedule is depicted in Figure 6, which evidently demonstrates its high effectiveness. The electricity price changes at different hours, but stays the same within one hour. The highest pricing peak appears in the evening from $7 \mathrm{pm}$ to $9 \mathrm{pm}$ on March-3, while the lowest pricing valley falls in the early morning from 3am to 7am on March-4. This scheduling solution can not only effectively avoid high-priced periods, e.g., the aforementioned highest pricing peak, but also allocate the jobs to low-priced periods as many as possible, e.g., from $4 \mathrm{pm}$ to $7 \mathrm{pm}$ on March-3 and the aforementioned pricing valley.

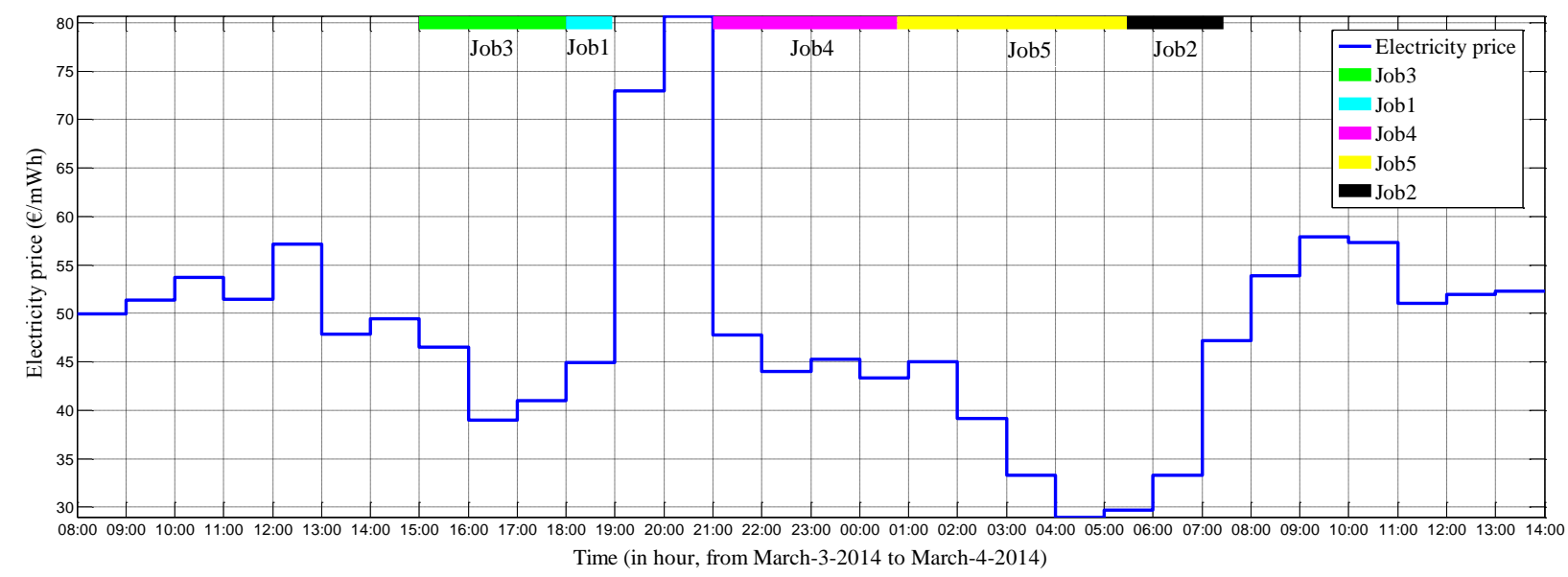

Figure 6 RTP electricity price from Belpex and the optimal job schedule (the scheduling step is 1 second)

This optimal schedule is further compared with some other cases (see Table 8). The maximum pricing takes the highest electricity price during the studied period and the maximal number of machine startup and shutdown. Similarly, the average pricing takes the mean values. As a classical production schedule, the as-early-as-possible schedule drives the machine to grind all the jobs consecutively from the beginning of the work shifts without staying idle or powered off between any jobs. In comparison, the backward schedule plans the start time of jobs from the due time. This leads to the as-late-as-possible schedule. These cases all follow the same job sequence of the optimal schedule. As is shown by Table 8 , the cost reduction effect of the optimal schedule is obvious with the cost saving rates varying from $7 \%$ to $52 \%$.

\begin{tabular}{llcll} 
Table 8 Comparison between the optimal job schedule and some theoretical cases \\
\hline Case & $\begin{array}{l}\text { Electricity price } \\
(€ / \mathrm{mWh})\end{array}$ & $\begin{array}{l}\text { Number of machine } \\
\text { startup and shutdown }\end{array}$ & $\begin{array}{l}\text { Cost }(€) \\
\text { Maximum pricing }\end{array}$ & $\begin{array}{l}\text { Percentage of cost } \\
\text { saving }\end{array}$ \\
Average pricing & 80.69 & 3 & 10.7 & $\mathbf{5 2 \%}$ \\
As-early-as-possible schedule & Hourly dynamic & 1 & 6.3 & $\mathbf{1 9 \%}$ \\
As-late-as-possible schedule & Hourly dynamic & 1 & 5.8 & $\mathbf{1 2 \%}$ \\
The optimal schedule & Hourly dynamic & 2 & 5.5 & $\mathbf{7 \%}$ \\
\hline
\end{tabular}

\subsubsection{Energy simulation of the scheduling solution}

As the scheduler is coupled with the energy model (see Section 4.1), detailed energy related statistics can be further given by the energy simulation of the optimal schedule, including the accumulated time duration, the electric consumption, and the cost at the level of machine states, and also the aggregated information at the machine level (see Table 9). The main electricity consumer among states can be identified as Grinding, which takes up nearly $80 \%$ of the total electric consumption and cost, followed by Dressing at nearly $20 \%$. This type of table enables machine operators and decision-makers to have a clear view over the energy related details of the machine. 
Table 9 Energy consumption details at levels of the machine and machine states for the optimal schedule

\begin{tabular}{|c|c|c|c|c|c|c|}
\hline \multirow{2}{*}{$\begin{array}{l}\text { Grinder } \\
\text { state }\end{array}$} & \multicolumn{2}{|c|}{ Time consumption } & \multicolumn{2}{|c|}{ Electric consumption } & \multicolumn{2}{|c|}{ Electric cost } \\
\hline & Amount (s) & Percentage $^{\mathrm{a}}$ & Amount (kWh) & Percentage $^{\mathrm{a}}$ & Amount $(€)$ & Percentage $^{\mathrm{a}}$ \\
\hline Off & 55172 & $51.1 \%$ & 0 & 0 & 0 & 0 \\
\hline Startup & 1304 & $1.2 \%$ & 1.29 & $1.0 \%$ & 0.08 & $1.6 \%$ \\
\hline Ready & 175 & $0.2 \%$ & 0.29 & $0.2 \%$ & 0.01 & $0.2 \%$ \\
\hline Grinding & 37500 & $34.7 \%$ & 98.85 & $79.0 \%$ & 3.97 & $78.5 \%$ \\
\hline Dressing & 13125 & $12.2 \%$ & 24.50 & $19.6 \%$ & 0.98 & $19.4 \%$ \\
\hline Unowned & 50800 & $47.0 \%$ & 123.64 & $98.8 \%$ & 4.96 & $98.0 \%$ \\
\hline Shutdown & 724 & $0.7 \%$ & 0.20 & $0.2 \%$ & 0.01 & $0.2 \%$ \\
\hline Grinder & $108000(30 \mathrm{~h})$ & - & 125.13 & - & 5.06 & - \\
\hline
\end{tabular}

In addition, the power consumption of this grinder during the simulation can be represented along the time course, as shown in Figure 7. The grinder's energy consumption states are correspondingly indicated above the power curve. Figure 7 is a high-precision visualization, since the presentation can be zoomed at one second, which is illustrated by the left arrow. There are in fact two power consumption curves: the green one indicates the a priori estimation, which externally draws a prediction curve only according to the given job schedule independent of the simulation environment, while the dark blue one is the a posteriori display based on the power data collected during the simulation. The perfect overlap of the two curves demonstrates the excellent functionality of the proposed model to conduct energy modeling and simulation. Given the production schedule and the electricity price in the coming days, this representation can also serve as an accurate power consumption prediction. Besides, the accurate power consumption behavior can be stored and compared with unrecognized power consumption patterns. This facilitates MFs to be detected in the early stage of abnormal events on a shop floor.

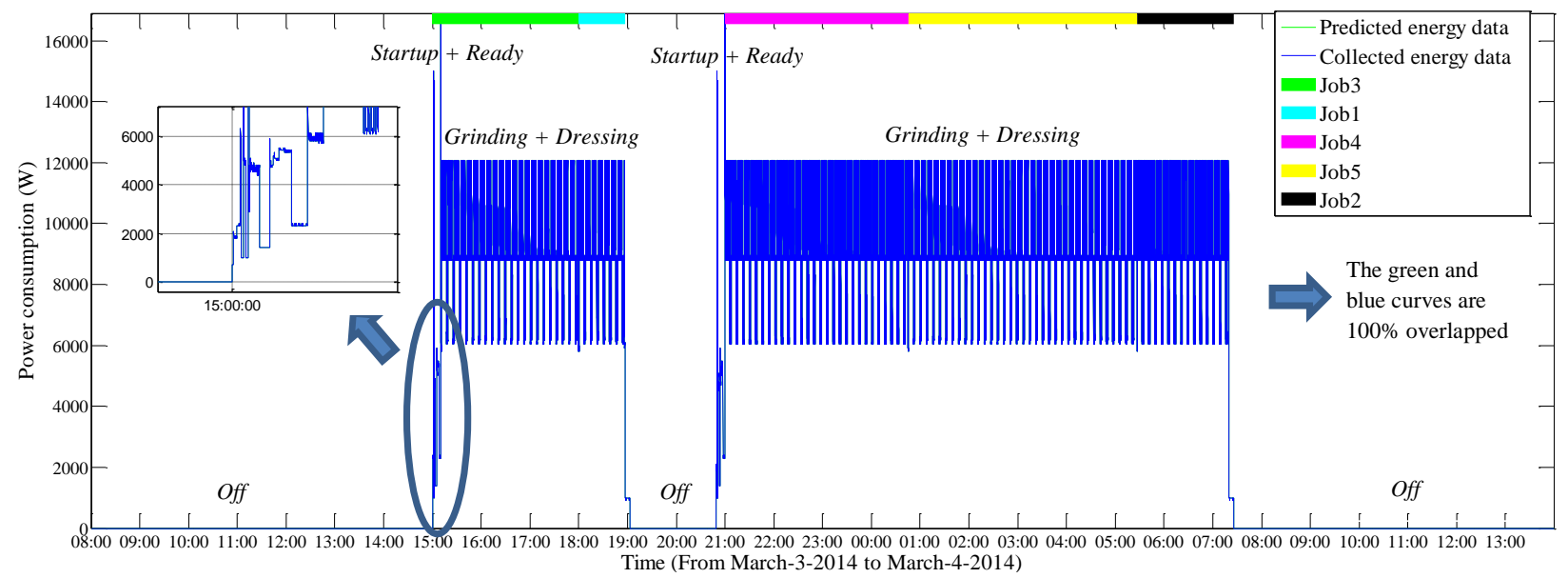

Figure 7 Visualization of the grinder's power consumption (the modeling step is 1 second)

\subsection{Single-machine job scheduling under time-of-use pricing (ToUP)}

The ToUP tariff was taken from a Belgian plastic bottle manufacturer, which buys energy from the spot market once a month. All the other assumptions are the same as those in the above case. In order to further demonstrate the effectiveness of the proposed energy-cost-aware scheduler, the investigated period is extended to one week, i.e., from 8am on March-3-2014 to 8am on March-10-2014. The job number rises to 35, by raising to 7 times, compared to the number of each job in Table 4. The scheduling step is one second, which is similar to the former case.

As depicted in Figure 8, this electricity price has two levels: on-peak and off-peak within every 24 hours, at $61.1 € / \mathrm{mWh}$ and $39.6 € / \mathrm{mWh}$, respectively. The off-peak period lasts from $9 \mathrm{pm}$ to 6 am of the next day, which has 
only nine hours within a day. Hence, the obtained job schedule makes use of these periods as many as possible, while keeping energy-related overheads as small as possible. The energy-related overheads can be extra energy consumed by a frequent machine switch on/off, or/and by a long-term machine idle state. In this obtained schedule, there are some short off-peak slots that are allocated for the idle state, instead of the production-related states, i.e., Grinding and Dressing, which are more energy intensive. This can be illustrated by the idle periods of $14 \mathrm{~m} 35 \mathrm{~s}$ between Job9 and Job11 during the Wednesday off-peak, and 1h3m20s between Job6 and Job34 during the Thursday off-peak. In total, $90 \%$ of the off-peak periods are allocated for production-related states. Besides, the machine operation, which is scheduled to follow each job, is indicated next to each job in the legend of Figure 8. This is similar to Table 7 in the RTP case. The machine operation will invoke the corresponding machine state transition in the state-based energy model. Coupled with the energy model, the scheduler thus assigns the proper machine states along with jobs.

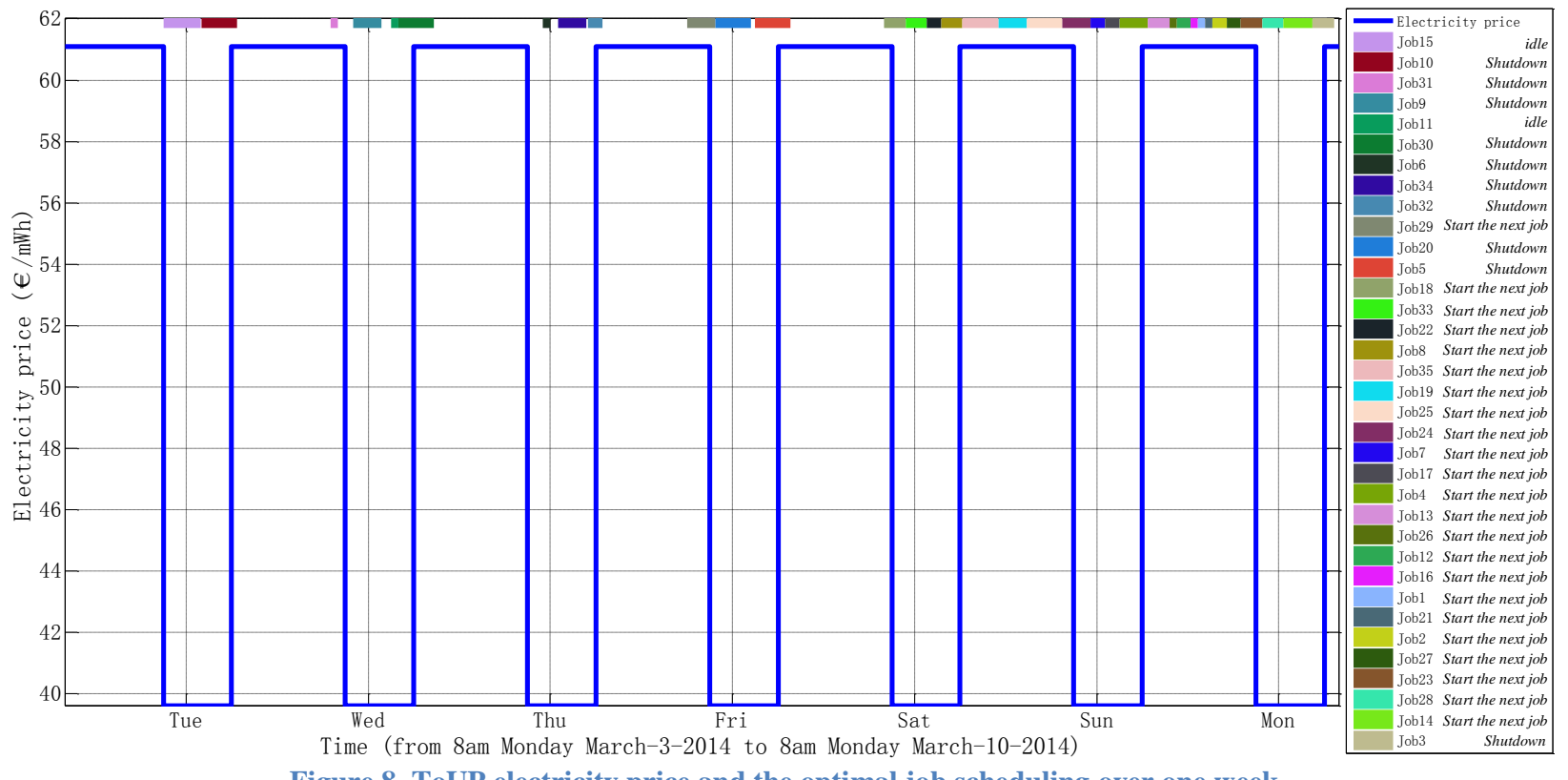

Figure 8 ToUP electricity price and the optimal job scheduling over one week

Therefore, the obtained schedule is revealed as a near-optimal solution, instead of the optimal solution. One key reason is that there are totally 604800 time slots in this demonstration. This turns out to be a large number for scheduling, in comparison with maximum dozens or hundreds of time slots in similar work (Liu et al., 2014; Shrouf et al., 2014). This near-optimal schedule is obtained after a 2593-second GA search. Figure 9 depicts this GA search trend. In the first 20 generations, the total electricity cost of the best scheduling solution decreases rapidly from $45.18 €$ to $42.86 €$. Then from the $21^{\text {st }}$ generation to the $67^{\text {th }}$ generation, the cost experiences a steady decrease down to $42.63 €$. After that, the cost stays quite stable with only a slight decrease, and reaches $42.61 €$ at the $250^{\text {th }}$ generation. Besides, Figure 8 and Figure 9 jointly demonstrate that the GA search approaches to the actual optimum.

Based on the energy simulation of the optimal job schedules in the two electric tariffs, a comparison is further conducted between their energy consumption efficiency $E F_{c n p t}$, EC efficiency $E F_{c t}$, and productive energy rate $R_{p}$ (see Table 10). $E F_{\text {cnpt }}$ indicates that, for producing one workpiece, the two optimal schedules consume almost the same amount of energy under RTP and ToUP, respectively. $E F_{c t}$ shows that, for one workpiece, it consumes a lower electric cost (17\%) under RTP than under ToUP. $R_{p}$ reveals the percentage of the consumed electricity which directly contributes to the added value of workpieces. It stays at the same rate $(79 \%)$ in the two cases, although the time duration and job quantity are different. This can be explained by two raisons. First, the grinder is scheduled to be powered off during most time periods when there is no need for grinding. Second, the dressing operation is accompanied with the grinding operation periodically. So the energy consumed by grinding and dressing increases proportionately along with the growth of job number. This type of table can not only provide machine energy related 
KPIs to decision-makers, but also help them to get an accurate insight into the effect of different electric tariffs on the energy related KPIs. Therefore, the knowledge of the energy consumption and EC contributes to a more informed decision on production activities at a shop floor.

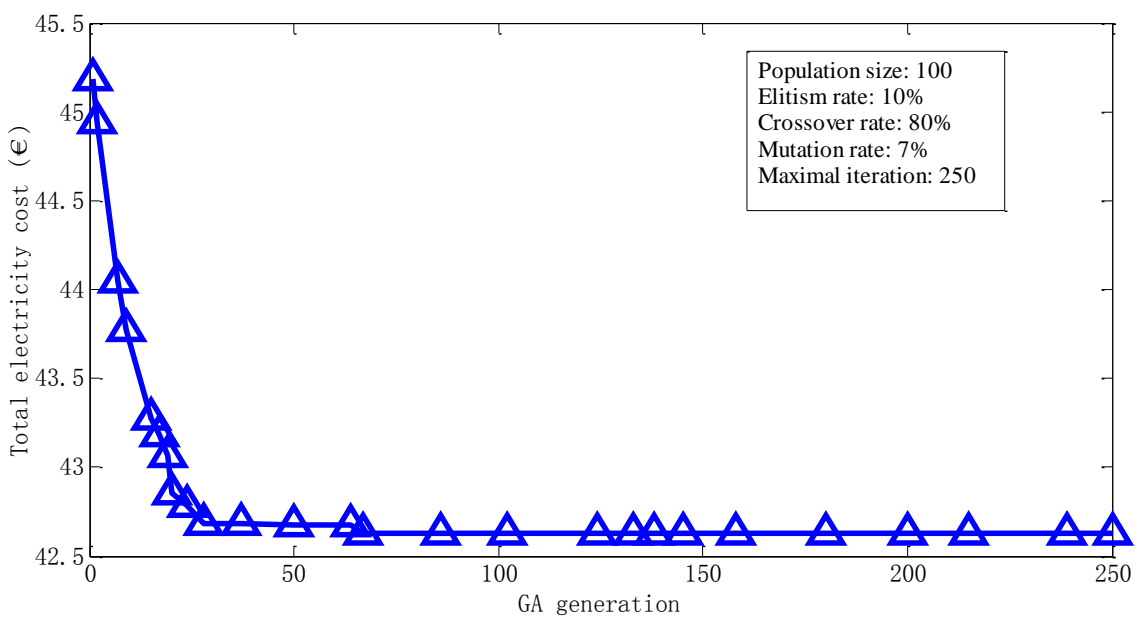

Figure 9 GA search trend

Table 10 Energy KPIs at different electric tariffs

\begin{tabular}{clc}
\hline & RTP tariff & ToUP tariff \\
\hline$E F_{\text {cnpt }}$ & $0.0834 \mathrm{kWh} / \mathrm{piece}$ & $0.0833 \mathrm{kWh} / \mathrm{piece}$ \\
$E F_{\text {cnpt }}=$ Total energy $/$ Total amount of products $)$ & & \\
$\begin{array}{c}\left.E F_{\text {ct }}=\text { Total energy cost } / \text { Total amount of products }\right) \\
R_{p}\end{array}$ & $0.0034 € /$ piece & $0.0041 € /$ piece \\
$\left(R_{p}=\right.$ Productive energy/Total energy $)$ & $79 \%$ & $79 \%$ \\
\hline
\end{tabular}

\subsection{Stochasticity}

Two types of SEs were implemented to demonstrate the effectiveness of stochasticity handling in the energycost-aware scheduling (see Section 3.4), and further to enable the analysis of how stochasticity affects the energycost-effective performance of the proposed method. The case of scheduling under RTP in Section 4.2 was taken as a baseline case. The first investigated type of SE is a random MF. The time for a MF follows the Weibull distribution, with the following probability density function (Johnson et al., 1994):

$$
f(t ; \alpha, \beta)= \begin{cases}\frac{\beta}{\alpha}\left(\frac{t}{\alpha}\right)^{\beta-1} e^{-\left(\frac{t}{\alpha}\right)^{\beta}} & x \geq 0 \\ 0 & x<0\end{cases}
$$

In this investigation, the shape parameter $\alpha$ equals one, meaning that the MF rate is constant along time; the scale parameter $\beta$ equals 10000 in order to adapt the time generation interval to the investigated period (30h, i.e., 108000s). There is one MF during an energy simulation of a complete schedule. The duration of each MF is one hour. The machine stays powered off during the MF period. At the presence of a MF, the interrupted job is resumable, and the rest of it should be place in the first place in the reschedule. Otherwise, it is not possible to separate the jobs.

As the baseline case is a fresh schedule, the scheduler has the same configuration, e.g., the job number and duration, RTP energy price, etc. It first goes through the heuristic steps "Start-O1-O4-O5" (see Figure 3) to get the original optimal schedule (see Figure 10). This optimal schedule is then simulated in O6. Upon the MF which occurs at $15 \mathrm{~h} 29 \mathrm{~m} 35 \mathrm{~s}$ on March-3-2014, the energy simulation terminates, and the scheduler continues to go through the steps "O7-O2-O3-O4-O5" (see Figure 3) to reschedule the order and start time of the upcoming jobs (i.e., Job5, Job1, Job2, and Job4, see Figure 10), and also to reschedule the start time of the non-executed part of Job3. As shown by 
Figure 10, the jobs are successfully rescheduled by making use of the low-priced periods and avoiding the highpriced periods. The total EC (5.2€) is then comprised of the cost for producing Job3's executed part in the original schedule, and the cost for running the whole reschedule. It slightly increases by $2 \%$ in comparison to the EC of the baseline case. The reason is that the MF takes up some low-priced periods, such that there is not fully sufficient lowpriced periods to accommodate the reschedule after the MF (e.g., the last part of Job3 after 19h on March-3-2014). The EC rising rate caused by the MF will get higher, if the EC consumed by the maintenance activity during the MF is considered.

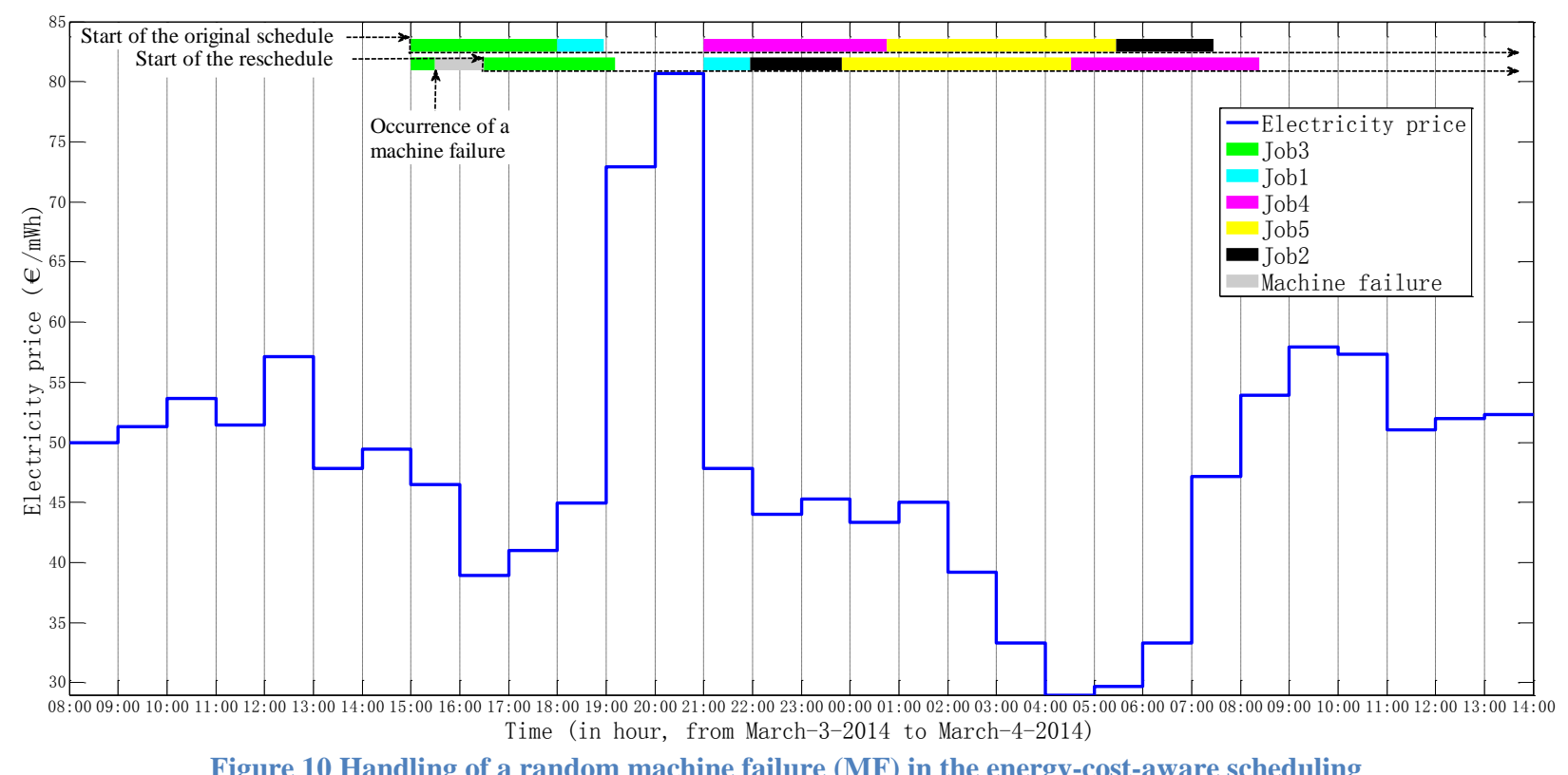

The second considered type of SE is a random increase of a customer order. Five new small jobs (see Table 11) arrive at $17 \mathrm{~h}$ on March-4, which is during the execution of Job3 in the original schedule. They have the same due time as the original jobs (i.e., $2 \mathrm{pm}$ on March-4-2014). The reschedule is thus triggered. Job3 continues to be executed, while all the upcoming jobs (the non-executed original jobs plus the new jobs numbered from 6 to 10) are rescheduled by going through the steps "O7-O2-O3-O4-O5-O6" (see Figure 3). The start time of the reschedule is the time when Job3 is finished. As presented by Figure 11, the rescheduled jobs effectively make use of the lowpriced periods, while avoiding the high-priced periods (i.e., $19 \mathrm{~h}$ to $21 \mathrm{~h}$ on March-3, and $9 \mathrm{~h}$ to $11 \mathrm{~h}$ on March-4). The total EC for all the jobs is $6.35 €$. Compared to the baseline case, it rises $25 \%$, while the number of workpieces increases $20 \%$. The reason why the $\mathrm{EC}$ has a higher increasing rate is that some higher-priced periods have to be used to accommodate the rising job volume. However, the difference between the two rising rates is relatively small (i.e., 5\%), in comparison to the large variation of the energy price around its mean level (i.e., 16\%). This further indicates the energy-cost-effectiveness of the proposed method.

The above two cases and any other SE handling in scheduling can be viewed as an online scheduling problem. In this problem, some of the information around the upcoming jobs is unknown when running the scheduler. This is illustrated by a random MF, which occurs during the original schedule. Information needs to be updated that the original jobs need to be scheduled outside the period of a MF. This is additionally illustrated by a later arrival of new jobs, which necessitates an update of the number and processing time of the upcoming jobs, even the type of jobs which may have different power consumption. Once a SE takes place, the cyclic steps "O7-O2-O3-O4-O5-O6" in Figure 3 actually serve as an effective approach to revise the original schedule, of which the part is not yet executed by the machine. The frequency to start the reschedule depends on the occurrence frequency of a SE. Since a shop floor is generally well maintained, the frequency should be low. Furthermore, the proposed method rapidly achieves a near-optimal scheduling solution (see Figure 9). As a result, computation time is not a critical factor to 
limit the scheduling performance. However, the propose method aims at a unit process. If it extends to multiple machines, multiple lines, and even multiple factories, computation time will impose increasingly evident constraints. Table 11 New grinding jobs for scheduling

\begin{tabular}{lccccc}
\hline \multicolumn{1}{c}{ Job ID } & 6 & 7 & 8 & 9 & 10 \\
\hline Number of steel workpieces & 80 & 70 & 60 & 50 & 40 \\
Required production time in & 2625 & 2375 & 2000 & 1625 & 1250 \\
seconds (grinding + dressing) & $(43 \mathrm{~m} 45 \mathrm{~s})$ & $(39 \mathrm{~m} 35 \mathrm{~s})$ & $(33 \mathrm{~m} 20 \mathrm{~s})$ & $(27 \mathrm{~m} 5 \mathrm{~s})$ & $(20 \mathrm{~m} 50 \mathrm{~s})$ \\
\hline
\end{tabular}

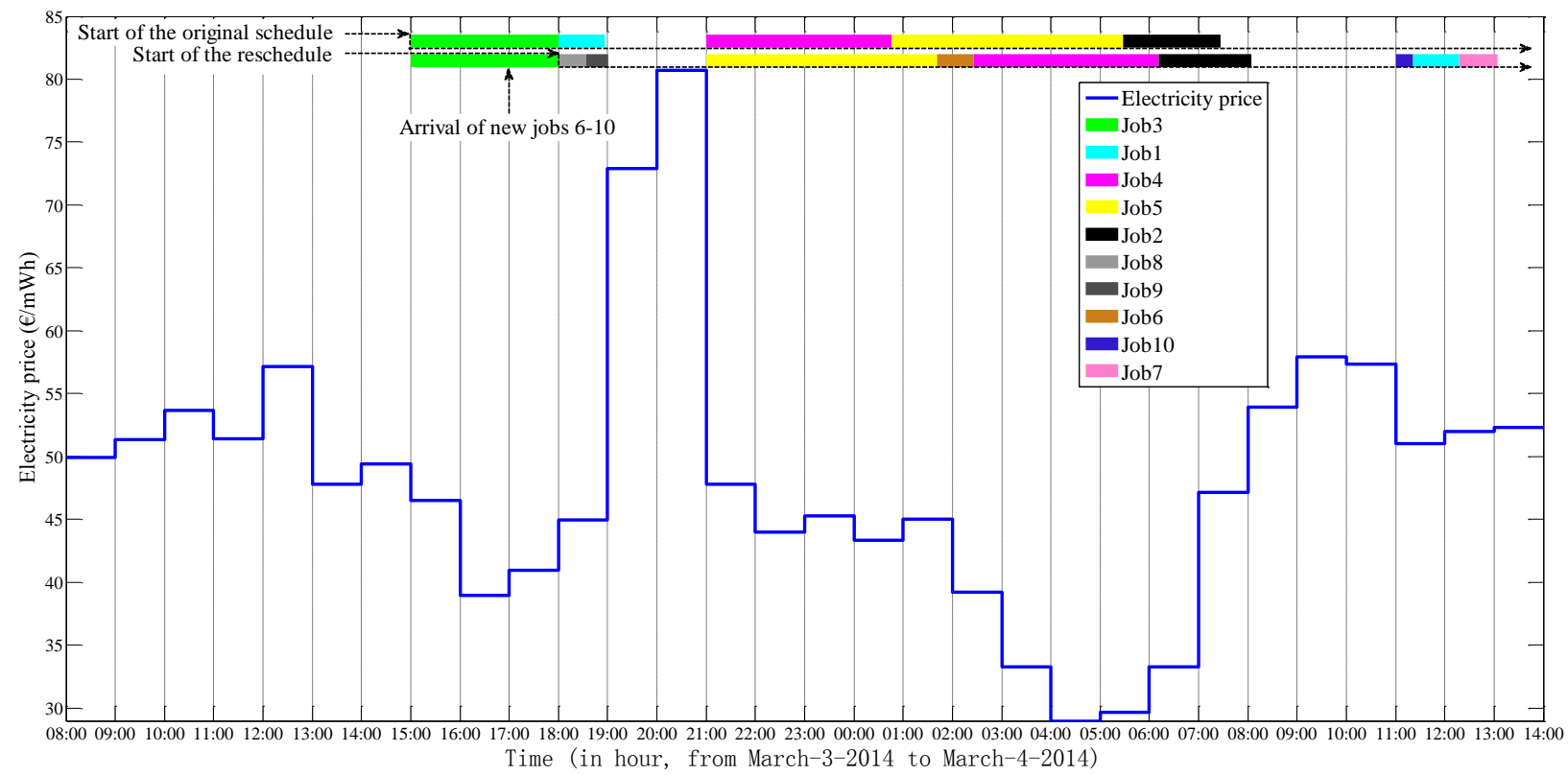

Figure 11 Handling of new jobs in the energy-cost-aware scheduling

\section{Conclusions}

A novel method has been proposed in this study to conduct energy-cost-aware job scheduling on a machine. Finite state machines (FSMs) are used to build the energy model of a machine, and to run the energy simulation which has an interface to dynamic energy prices. Coupled with the energy model, a mixed-integer linear programming (MILP) model is formulated for scheduling jobs on a single machine. A genetic algorithm (GA) is implemented in Java, in order to search for the optimal or near-optimal scheduling solution at volatile energy prices. By coupling the energy modeling and the energy-aware scheduling, this method jointly combines the energy efficiency and demand response efforts within the demand side management (DSM) framework. This fills the gap in the literature which simultaneously encompasses energy modeling, simulation, and optimization of single production processes at the level of machine states.

This method was successfully applied to a numeric control grinding machine (Paragon RC-18CNC). First, a state-based energy model was built with the power data measured from this machine. It was validated by means of simulation. With regard to industrial applications, it is an effective way to use the large amount of energy data, which is collected either by temporal measurement setups or by long-term monitoring systems. It can provide energy managers with energy and cost information at levels of machine states and a single machine. It thus unveils the energy consumption behaviors of machines, and facilitates measures for enhancing machine energy efficiency.

Second, the effectiveness of the scheduler was demonstrated under real-time pricing (RTP) and time-of-use pricing (ToUP) tariffs, over different time periods (i.e., 30 hours and 7 days), and at a time step of one second. This scheduler allocates jobs to lower-priced periods without causing energy overheads, and assigns complete machine states over time, in order to minimize the involving energy cost (EC) within a given due time. On the one hand, 
obvious economic savings may be thus achieved for the industry. On the other hand, this may help power generators to take advantage of sustainable energy systems by keeping industrial energy demand within the provision capacity of renewable energy sources, which stimulates the reduction of the carbon footprint in the environment.

Third, the proposed scheduler highly interacts with the state-based energy model. Therefore, the scheduler can not only get detailed energy information as its input, but also output the searched schedule for detailed energy simulation. As the schedule plans for the future production, the energy consumption of a single machine can be further forecasted based on the optimal production schedule. The forecasted energy information at levels of states and a machine under different electric tariffs may facilitate an enterprise to carry on an improved energy contact negotiation with the utility. As a result, the utility can calculate a more accurate demand prognosis, meanwhile the enterprise will have the possibility to get from the utility a more customizable energy provision and a lower EC.

Fourth, stochasticity is generally considered in the proposed method. According to the investigation, a random machine failure tends to be harmful. It not only impedes the normal production activity, but also forms up a competitive relationship with the energy-cost-aware scheduler in regard to taking up low-priced periods. Nevertheless, stochasticity is demonstrated to be well handled by the proposed method, such that its harmful influence on the EC effectiveness can be minimized. Besides, new jobs, which arrive during an ongoing execution of a schedule, may have an influence on the scheduling of all the upcoming jobs along the volatile energy price. The upcoming jobs are demonstrated to be effectively rescheduled, such that the total EC is minimized.

Future work will include an extension to a multi-criteria scheduler coupled with the energy model. This scheduler considers optimizing several important performance indicators of a machine, such as makespan, energy consumption, carbon emissions etc. A more flexible energy modeling can also be considered with more machine parameters and measurements to widely cover the energy consumption behaviors of a machine. If the proposed approach extends to a larger scale, e.g., multiple machines, lines, and factories, a meaningful solution is envisioned to be obtained for energy-efficient and energy-cost-effective production in a general sense.

\section{Acknowledgement}

This research was supported by the SENCOM project (More info at http://www.iminds.be/en/projects/2014/03/20/sencom). The SENCOM project is co-funded by iMinds (Interdisciplinary institute for Technology), a research institute founded by the Flemish Government. Special thanks are given to the company System Insights for providing measured power data.

\section{Reference}

Abele, E., Eisele, C., Schrems, S., 2012. Simulation of the energy consumption of machine tools for a specific production task, in: Dornfeld, D.A., Linke, B.S. (Eds.), Leveraging technology for a sustainable world. Springer Berlin Heidelberg, pp. 233-237.

Ajorlou, S., Shams, I., 2013. Artificial bee colony algorithm for conwip production control system in a multi-product multimachine manufacturing environment. J Intell Manuf 24, 1145-1156.

Babu, C.A., Ashok, S., 2008. Peak load management in electrolytic process industries. Power Systems, IEEE Transactions on 23, 399-405.

Belpex, 2013. Belpex brochure. http://www.belpex.be/wp-content/uploads/APX1318_ProductSheet_Belpex_final_LR.pdf (access 01.08.14).

Belpex, 2014. Belgium power exchange. http://www.belpex.be/ (accessed 29.07.14).

Cardenas, J.A., Gemoets, L., Ablanedo Rosas, J.H., Sarfi, R., 2014. A literature survey on smart grid distribution: An analytical approach. Journal of Cleaner Production 65, 202-216.

Chiarini, A., 2014. Sustainable manufacturing-greening processes using specific lean production tools: An empirical observation from european motorcycle component manufacturers. Journal of Cleaner Production 85, 226-233.

Chien, C.-F., Hsu, C.-Y., Hsiao, C.-W., 2012. Manufacturing intelligence to forecast and reduce semiconductor cycle time. J Intell Manuf 23, 2281-2294.

Cui, W.-W., Lu, Z., Pan, E., 2014. Integrated production scheduling and maintenance policy for robustness in a single machine. Computers \& Operations Research 47, 81-91.

Davito, B., Tai, H., Uhlaner, R., 2010. The smart grid and the promise of demand-side management. 38-44. https://www.smartgrid.gov/sites/default/files/doc/files/The Smart Grid Promise DemandSide Management 201003.pdf (accessed 29.07.14). 
Devoldere, T., Dewulf, W., Deprez, W., Duflou, J., 2008. Energy related life cycle impact and cost reduction opportunities in machine design: The laser cutting case, 15th CIRP International Conference on Life Cycle Engineering: Conference Proceedings Sydney, N.S.W., pp. 412-419.

Diaz, N., Redelsheimer, E., Dornfeld, D., 2011. Energy consumption characterization and reduction strategies for milling machine tool use, in: Hesselbach, J., Herrmann, C. (Eds.), Glocalized solutions for sustainability in manufacturing. Springer Berlin Heidelberg, pp. 263-267.

Dietmair, A., Verl, A., 2009a. Energy consumption forecasting and optimization for tool machines. Modern Machinery Science Journal, 62-67.

Dietmair, A., Verl, A., 2009b. A generic energy consumption model for decision making and energy efficiency optimisation in manufacturing. International Journal of Sustainable Engineering 2, 123-133.

Eberspächer, P., Schraml, P., Schlechtendahl, J., Verl, A., Abele, E., 2014. A model- and signal-based power consumption monitoring concept for energetic optimization of machine tools. Procedia CIRP 15, 44-49.

Fang, K.-T., Lin, B.M.T., 2013. Parallel-machine scheduling to minimize tardiness penalty and power cost. Computers \& Industrial Engineering 64, 224-234.

Gelazanskas, L., Gamage, K.A.A., 2014. Demand side management in smart grid: A review and proposals for future direction. Sustainable Cities and Society 11, 22-30.

Gutowski, T., Dahmus, J., Thiriez, A., 2006. Electrical energy requirements for manufacturing processes, Proceedings of the 13th CIRP International Conference on Life Cycle Engineering, Leuven, Belgium, pp. 623-628.

Hadera, H., Harjunkoski, I., Sand, G., Grossmann, I.E., Engell, S., 2015. Optimization of steel production scheduling with complex time-sensitive electricity cost. Computers \& Chemical Engineering 76, 117-136.

He, Y., Li, Y., Wu, T., Sutherland, J.W., 2015. An energy-responsive optimization method for machine tool selection and operation sequence in flexible machining job shops. Journal of Cleaner Production 87, 245-254.

Huang, B., Jiang, R., Zhang, G., 2014. Search strategy for scheduling flexible manufacturing systems simultaneously using admissible heuristic functions and nonadmissible heuristic functions. Computers \& Industrial Engineering 71, 21-26.

Jang, D., Eom, J., Kim, M.G., Rho, J.J., 2015. Demand responses of korean commercial and industrial businesses to critical peak pricing of electricity. Journal of Cleaner Production 90, 275-290.

Johnson, N.L., Kotz, S., Balakrishnan N. 1994. Continuous Univariate Distributions, vol. 1, second ed. John Wiley \& Sons, Inc.

Kellens, K., Dewulf, W., Overcash, M., Hauschild, M., Duflou, J., 2012a. Methodology for systematic analysis and improvement of manufacturing unit process life-cycle inventory (UPLCI) - CO2PE! Initiative (cooperative effort on process emissions in manufacturing). Part 1: Methodology description. Int J Life Cycle Assess 17, 69-78.

Kellens, K., Dewulf, W., Overcash, M., Hauschild, M., Duflou, J., 2012b. Methodology for systematic analysis and improvement of manufacturing unit process life cycle inventory (UPLCI) CO2PE! Initiative (cooperative effort on process emissions in manufacturing). Part 2: Case studies. Int J Life Cycle Assess 17, 242-251.

Küster, T., Lützenberger, M., Freund, D., Albayrak, S., 2013. Distributed evolutionary optimization for electricity price responsive manufacturing using multi-agent system technoloy. International Journal on Advances in Intelligent Systems 6 , 27-40.

Li, W., Kara, S., Kornfeld, B., 2013. Developing unit process models for predicting energy consumption in industry: A case of extrusion line, in: Nee, A.Y.C., Song, B., Ong, S.-K. (Eds.), Re-engineering manufacturing for sustainability. Springer Singapore, pp. 147-152.

Liu, Y., Dong, H., Lohse, N., Petrovic, S., 2015. Reducing environmental impact of production during a rolling blackout policy a multi-objective schedule optimisation approach. Journal of Cleaner Production 102, 418-427.

Liu, Y., Dong, H., Lohse, N., Petrovic, S., Gindy, N., 2014. An investigation into minimising total energy consumption and total weighted tardiness in job shops. Journal of Cleaner Production 65, 87-96.

Lu, S.-M., Lu, C., Tseng, K.-T., Chen, F., Chen, C.-L., 2013. Energy-saving potential of the industrial sector of taiwan. Renewable and Sustainable Energy Reviews 21, 674-683.

Luo, H., Du, B., Huang, G.Q., Chen, H., Li, X., 2013. Hybrid flow shop scheduling considering machine electricity consumption cost. International Journal of Production Economics 146, 423-439.

Mechs, S., Muller, J.P., Lamparter, S., Peschke, J., 2012. Networked priced timed automata for energy-efficient factory automation, American Control Conference (ACC), 2012, pp. 5310-5317.

O'Driscoll, E., O'Donnell, G.E., 2013. Industrial power and energy metering - a state-of-the-art review. Journal of Cleaner Production 41, 53-64.

Pechmann, A., Schöler, I., Hackmann, R., 2012. Energy efficient and intelligent production scheduling - evaluation of a new production planning and scheduling software, in: Dornfeld, D.A., Linke, B.S. (Eds.), Leveraging technology for a sustainable world. Springer Berlin Heidelberg, pp. 491-496.

Shrouf, F., Miragliotta, G., 2015. Energy management based on internet of things: Practices and framework for adoption in production management. Journal of Cleaner Production, in press.

Shrouf, F., Ordieres-Meré, J., García-Sánchez, A., Ortega-Mier, M., 2014. Optimizing the production scheduling of a single machine to minimize total energy consumption costs. Journal of Cleaner Production 67, 197-207.

Vikhorev, K., Greenough, R., Brown, N., 2013. An advanced energy management framework to promote energy awareness. Journal of Cleaner Production 43, 103-112.

Wang, Y., Li, L., 2013. Time-of-use based electricity demand response for sustainable manufacturing systems. Energy 63, 233244. 
Weinert, N., Mose, C., 2014. Investigation of advanced energy saving stand by strategies for production systems. Procedia CIRP $15,90-95$.

Zhang, H., Zhao, F., Fang, K., Sutherland, J.W., 2014. Energy-conscious flow shop scheduling under time-of-use electricity tariffs. CIRP Annals - Manufacturing Technology 63, 37-40. 